This item was submitted to Loughborough's Research Repository by the author.

Items in Figshare are protected by copyright, with all rights reserved, unless otherwise indicated.

\title{
Classification of human hand movements using surface EMG for myoelectric control
}

PLEASE CITE THE PUBLISHED VERSION

http://dx.doi.org/10.1007/978-3-319-46562-3_22

PUBLISHER

Springer

VERSION

AM (Accepted Manuscript)

\section{PUBLISHER STATEMENT}

This work is made available according to the conditions of the Creative Commons Attribution-NonCommercialNoDerivatives 4.0 International (CC BY-NC-ND 4.0) licence. Full details of this licence are available at: https://creativecommons.org/licenses/by-nc-nd/4.0/

\section{LICENCE}

CC BY-NC-ND 4.0

\section{REPOSITORY RECORD}

Wei, Jiefei, Qinggang Meng, and Atta Badii. 2019. "Classification of Human Hand Movements Using Surface EMG for Myoelectric Control”. figshare. https://hdl.handle.net/2134/24517. 


\title{
Classification of Human Hand Movements Using Surface EMG for Myoelectric Control
}

Jiefei Wei, Qinggang Meng, Atta Badii

\begin{abstract}
Surface electromyogram (sEMG) is a bioelectric signal that can be captured non-invasively by placing electrodes on the human skin. The sEMG is capable of representing the action intent of nearby muscles. The research of myoelectric control using sEMG has been primarily driven by the potential to create human-machine interfaces which respond to users intentions intuitively. However, it is one of the major gaps between research and commercial applications that there are rarely robust simultaneous control schemes. This paper proposes one classification method and a potential real-time control scheme. Four machine learning classifiers have been tested and compared to find the best configuration for different potential applications, and non-negative matrix factorisation has been used as a pre-processing tool for performance improvement. This control scheme achieves its highest accuracy when it is adapted to a single user at a time. It can identify intact subjects hand movements with above $98 \%$ precision and $91 \%$ upwards for amputees but takes double the amount of time for decision-making.
\end{abstract}

\section{Introduction}

Myoelectric control started to gain attention as a feature control mechanism in the 1940s [5]. It has the potential to lead a revolution in human-machine interaction due to its ability to measure human motion intention [5]. After more than 60 years of

Jiefei Wei

Loughborough University, Loughborough, UK, e-mail: J.Wei3-12@student.lboro.ac.uk

Qinggang Meng

Loughborough University, Loughborough, UK, e-mail: q.meng@lboro.ac.uk

Atta Badii

University of Reading, Reading, UK, e-mail: atta.badii@ reading.ac.uk 
research, however, myoelectric control is still struggling with the translation from research to clinical and commercial applications, such as exoskeletons, robot teleoperation, human-computer interface, and prostheses $[12,4,10]$. One of the major gaps between scientific research and common usage is the lack of robust simultaneous control schemes [11].

As described by Kuiken, T. A. et al., excellent results have been achieved by invasive methods for real-time myoelectric control of multifunction artificial arms [6]. On the other hand, non-invasive studies usually give an average classification accuracy of hand movements of up to 80-90\% [9]. Compared with invasive methods, non-invasive techniques are easier to set up and be accepted by users. Meanwhile, they do not impact on the human body.

Electromyogram(EMG) signals are bioelectrical signals generated during muscle activities, and surface EMG (sEMG) signals are those captured non-invasively through electrodes that placed on the skin. sEMG contains sufficient information about the individuals motion strength, orientation, and action type [1]. In this paper, the sEMG signals are used as the reference data for hand movement classification.

The paper is organised as follows: The benchmark database is described in Section 2. Then, Section 3 explains the algorithm signal pre-processing. The experimental results and analyses are presented in Section 4. In Section 5, a potential real-time control scheme is proposed. The paper is concluded in Section 6.

\section{NinaPro Database}

The NinaPro Database ( http://ninapro.hevs.ch/) is a large database containing the sEMG signal data acquired from 67 intact subjects and 11 hand-amputated subjects during different hand/arm actions [1]. It provides a good benchmark for EMG-based movement pattern interpretation, action classification and movement intention prediction. The NinaPro Database includes three sub-databases: Database 1, Database 2 and Database 3. Exercise B of Database 2 and Database 3 are used in this paper. Database 2 contains the sEMG acquired from 40 intact subjects, and Database 3 has sEMG signals acquired from 11 amputees. As illustrated in figure 1, Exercise B includes 17 diverse hand movements. The sEMG signals of Database 2 and Database 3 were captured by 12 Trigno Wireless electrodes (Deslys, Inc., www.delsys.com). "Eight electrodes are equally spaced around the forearm at the height of the radiohumeral joint; two electrodes are placed on the main activity spots of the flexor digitorum superficialis and the extensor digitorium superficialis; another two electrodes were put on the main activity spots of the biceps brachii and the triceps brachii [8]." Each movement is repeated six times by every subject. Each repetition lasted five seconds and was alternated with a resting posture lasting three seconds [8].

The original sEMG signals datasets from NinaPro are data matrices with $\mathrm{N}$ rows and 12 columns. Each column is a data channel that stores the continuously data captured by one Trigno electrode. Each row is a data vector that stores the data captured by 12 electrodes at one time. The sEMG signals are sampled at a rate of 
$2 \mathrm{KHz}$ with a baseline noise of less than $750 \mathrm{n} \mathrm{nV}$ RMS. Therefore, every row of raw data was captured at 0.5 milliseconds.

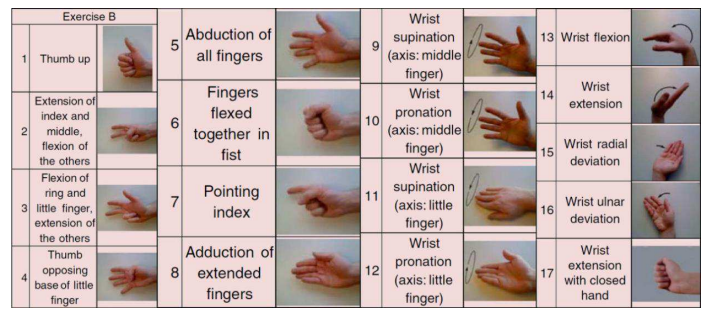

Fig. 1: Hand movements of Exercise B [8]

\section{Data processing and feature extraction}

sEMG signals are non-stationary, having complicated time, frequency, and timefrequency features; therefore, the raw data cannot be used as the input for classifier training; an appropriate data pre-processing is often more influential than the choice of classifiers [2, 3].

At the beginning of signal pre-treatment, a full-wave rectification is applied to convert the negative values to positive; so the non-negative matrix factorisation can be performed in the future. Then, the datasets need to be segmented into small data windows. The data window size should be short enough to reduce the decisionmaking time for intuitively simultaneous control. The data segments are not continuous; every data window has overlapped with the previous one. For example, if the first segment is from row 1 to 400; then the second segment will be from row 101 to 500. The overlapping can prevent feature loss during segmentation. For instance, there is a vital sEMG feature appearing from row 350 to 450 , if the data windows size is 400 rows without overlapping, then this feature will be fragmented by segmentation.

Feature extraction is performed based on the previous steps. There are two types of features in the sEMG signals; these are EMG features and Muscle Synergy features. "EMG features extract structural characteristics from a single channel to describe the specific signal [5]." The features of muscle synergy are time-invariant, containing information from multiple EMG channels that can depict the underlying muscle coordination principles while performing various motions [5]. Root mean square (RMS) is selected as the EMG feature from the datasets. The RMS is calculated segment by segment and channel by channel, then, each data window is

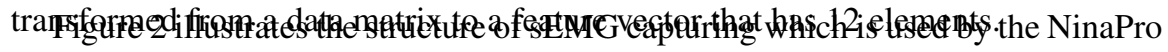
database. Twelve electrodes were used to generate the twelve channels datasets. However, there are more than twelve muscles in the human arm cooperating to support hand movements. Therefore, cross-talk is inevitable. The electrodes do not only 


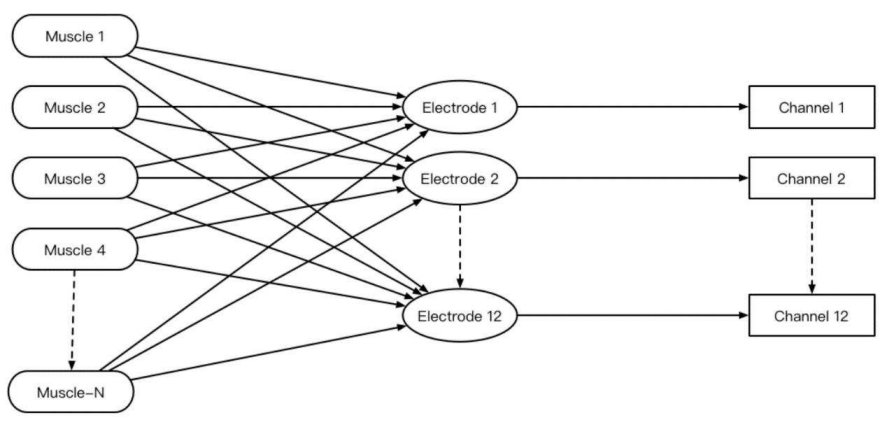

Fig. 2: Structure of sEMG capturing

capture the EMG signals from the targeted muscles but also catch the activities from all muscles that are involved in the same motion. Moreover, there are deep muscles that generate EMG signals which cannot be detected by surface electrodes directly, but these muscles make a significant contribution to hand motions. Therefore, non-negative matrix factorisation (NMF) has been applied to determine the original EMG signals of all the relevant muscles from the SEMG signals, for enhancing the performance of this classification scheme. NMF is a significant and expressive technique for blind source separation, which is capable of extracting original signals from the observed signals by way of an inverted mixing system. According to biology and anatomy, there are 24 muscles in the human arm. Thus, NMF has been used to expand the RMS feature matrix from 12 dimensions to 24 dimensions.

$$
X \cong W \times H+E
$$

Where $X$ is the $N \times 12$ RMS feature matrix of observed sEMG signals ( $N$ is the number of EMG data samples captured by 12 electrodes, $N \geq 1$ ). W is the $N \times 24$ feature matrix of source EMG signals from 24 muscles, and $\mathrm{H}$ is the $24 \times 12$ mixing matrix which represents the underlying muscles cooperation principles. $\mathrm{E}$ is the error or noise, that can be reduced by multiplicative update rules described by Daniel D. Lee and H.Sebastian Seung [7]. The following update rule was used in this study.

$$
H_{a u}=H_{a u} \frac{\left(W^{T} X\right)_{a u}}{\left(W^{T} W H\right)_{a u}} \quad W_{i a}=W_{i a} \frac{\left(X H^{T}\right)_{i a}}{\left(W H H^{T}\right)_{i a}}
$$

Hau and Wia represent one value of matrix $H$ and $W$ with au and ia as its coordinate. Once the NMF obtains the mixing matrix $H$, the feature matrix of source EMG signals that is used for classification can be calculated by

$$
S=H^{+} X
$$

Where $S$ is the feature matrix of source EMG signals, $H^{+}$is the Moore-Penrose pseudoinverse of mixing matrix $H$, and $X$ is the RMS feature matrix of observed sEMG signals. After signals pre-processing, four widely used classifiers: Support 
Vector Machines (SVM), Artificial Neural Networks (ANN), K-nearest neighbours (K-NN), and Adaboosted RandomForest (Ada-RF), have been trained and tested using the data from eight intact subjects and six amputees. The results from several configurations and their analyses are provided in the next section.

\section{Results and Analyses}

Table 1: Intact subjects basic information. RH: right handed; LH: left handed

\begin{tabular}{|l|l|l|l|l|l|l|l|l|}
\hline Subjects & 1 & 2 & 4 & 11 & 13 & 14 & 22 & 25 \\
\hline Gender & Male & Male & Female & Female & Male & Female & Female & Male \\
\hline Laterality & RH & RH & LH & RH & LH & RH & LH & LH \\
\hline Age & 29 & 29 & 30 & 33 & 30 & 30 & 28 & 30 \\
\hline Hight & $187 \mathrm{~cm}$ & $183 \mathrm{~cm}$ & $154 \mathrm{~cm}$ & $150 \mathrm{~cm}$ & $182 \mathrm{~cm}$ & $173 \mathrm{~cm}$ & $162 \mathrm{~cm}$ & $186 \mathrm{~cm}$ \\
\hline Weight & $75 \mathrm{~kg}$ & $75 \mathrm{~kg}$ & $50 \mathrm{~kg}$ & $54 \mathrm{~kg}$ & $70 \mathrm{~kg}$ & $59 \mathrm{~kg}$ & $54 \mathrm{~kg}$ & $90 \mathrm{~kg}$ \\
\hline
\end{tabular}

The basic information about the eight intact subjects is illustrated in Table 1. Figure 3 illustrates the classification results of eight intact subjects based on the RMS feature (before NMF) from four classifiers with a 400 sample segment size and 350 samples overlapping between two neighbouring segments. All the accuracy results are generated individually by ten fold cross-validation based on every subjects personal sEMG data, which means the classifiers that were trained on one specific users data can only identify that users movements accurately. Because the inputs for training and testing are the feature vectors that were calculated from the data segments, the classification precision represents the ability of how well the classifier can identify each data segment. Figure 4 shows the classification results of eight intact subjects based on the NMF feature; the rest of the configurations are as the same as Figure 3.

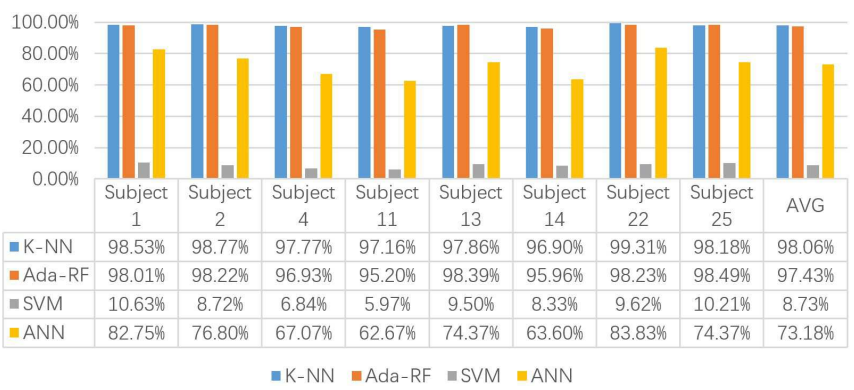

Fig. 3: Classification accuracy of intact subjects using RMS 
When using RMS feature, it is obvious that the K-NN and Ada-RF outperformed the SVM and ANN. The K-NN and Ada-RF reach $98.06 \%$ and $97.43 \%$ average precision of eight intact subjects. The results from ANN are 25\% lower than the K-NN and Ada-RF. The worst classifier is SVM, which gives an $8.73 \%$ average accuracy.

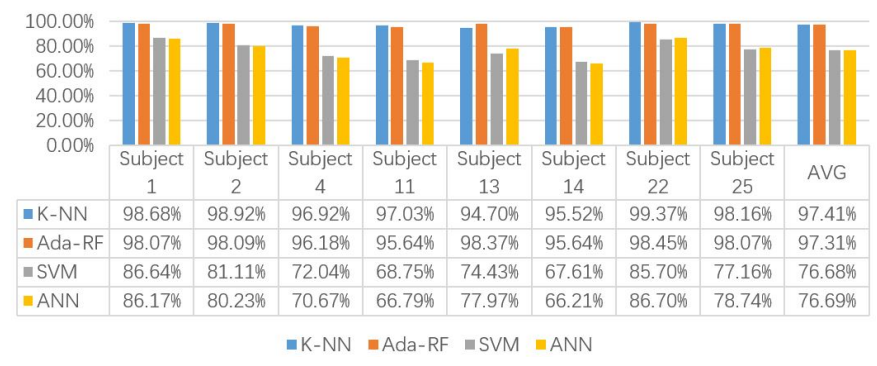

Fig. 4: Classification accuracy of intact subjects using NMF

The performance of SVM has been improved by NMF significantly from $8.73 \%$ to $76.68 \%$, and the accuracy of ANN is also increased by $3.51 \%$ using NMF. The K-NN and Ada-RF are still the best classifiers after applying NMF to the RMS feature.

For K-NN and Ada-RF, the NMF can improve the classification accuracy of subject 1 , subject 2 , subject 13 and subject 22 . However, the classification accuracy of subject 4 . subject 11, subject 14 and subject 25 have been impaired by NMF. Because the K-NN and Ada-RF outperform the SVM and ANN, the following tests results are based on K-NN and Ada-RF. Moreover, the performance of NMF is unpredictable for K-NN and Ada-RF, and it is also very time-consuming, the RMS feature is used for the following study.

The segment size and overlapping size can have a significant influence on classification accuracy. Table 2 shows the average accuracy of different overlapping sizes with fixed segment size. Moreover, Table 3 shows the mean accuracy of different segment sizes with fixed sample size of 50 moving forward step size. The test data proved the theory that is described in section 3, overlapping between segments can prevent feature loss, therefore, improving the classification accuracy. On the other hand, the increase in the segment size can also improve the classification accuracy. However, the segment size needs to be as small as possible to minimise the decision-making time. Based on the test data, 400 samples segment size with $87.5 \%$ overlapping ratio is the ideal choice for intact subjects.

Table 2: Classification accuracy against Overlapping size

\begin{tabular}{|c|c|c|c|c|c|}
\hline Overlapping size & $0 \%$ & $25 \%$ & $50 \%$ & $75 \%$ & $87.5 \%$ \\
\hline
\end{tabular}

\begin{tabular}{l|l|l|l|l|l|l|}
\hline Accuracy & $71 \%$ & $76 \%$ & $84 \%$ & $94 \%$ & $98 \%$ \\
\hline
\end{tabular}


Table 3: Classification accuracy against segment size

\begin{tabular}{|c|c|c|c|c|}
\hline Segment size & 200 samples & 400 samples & 800 samples & 1000 samples \\
\hline Accuracy & $91 \%$ & $98 \%$ & $99.5 \%$ & $99.8 \%$ \\
\hline
\end{tabular}

Precision is not the only key measurement of a classification scheme, the ability to adapt to a new user is also critical. As mentioned in section 2, every movement has six repeats. Figure 5 shows the average accuracy of K-NN and Ada-RF for intact subjects against how many repeats of sEMG data were used for training. Three repeats might be the best choice when quick adaptation is required, and the K-NN is the best classifier for intact subjects.

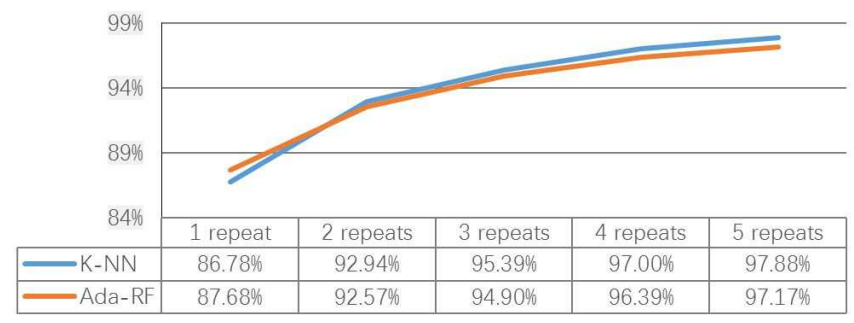

Fig. 5: Classification precision against training data quantity

Figure 6 illustrates the classification results of six amputees with two data window sizes by using a K-NN classifier. The 400 sample segment size cannot give good results especially for Amputee 7 who has no forearm. This issue can be solved by increasing the segment size to 800 samples, but the time for decision-making will also become twice as long since the window size doubled.

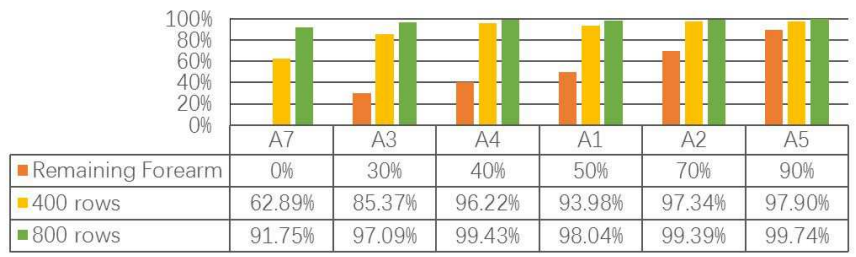

Fig. 6: classification accuracy of amputees with two segment size 


\section{Real-time Control Scheme}

The classification accuracy based on each single feature vector is excellent, but it is not reliable to just use one feature vector for decision-making in real-time control. The proposed decision-making unit is the three newest input feature vectors according to the computational power of the real devices.

Table 4: Decision-making matrix

\begin{tabular}{|l|l|l|l|l|l|l|l|l|}
\hline Vector 1 & $\mathrm{F}$ & $\mathrm{F}$ & $\mathrm{F}$ & $\mathrm{T}$ & $\mathrm{T}$ & $\mathrm{T}$ & $\mathrm{T}$ & $\mathrm{F}$ \\
\hline Vector 2 & $\mathrm{F}$ & $\mathrm{F}$ & $\mathrm{T}$ & $\mathrm{F}$ & $\mathrm{T}$ & $\mathrm{T}$ & $\mathrm{F}$ & $\mathrm{T}$ \\
\hline Vector 3 & $\mathrm{F}$ & $\mathrm{T}$ & $\mathrm{F}$ & $\mathrm{F}$ & $\mathrm{T}$ & $\mathrm{F}$ & $\mathrm{T}$ & $\mathrm{T}$ \\
\hline Decision & $\mathrm{F}$ & $\mathrm{F}$ & $\mathrm{F}$ & $\mathrm{F}$ & $\mathrm{T}$ & $\mathrm{T}$ & $\mathrm{T}$ & $\mathrm{T}$ \\
\hline
\end{tabular}

A decision matrix is created in Table 4. There are eight patterns during decisionmaking based on three feature vectors. If a feature vector has been classified correctly or a decision is made correctly, it has been marked as $\mathrm{T}$ with the colour green, else it is marked as $\mathrm{F}$ with the colour red. The decision-making unit will give a wrong answer if more than one feature vector has been classified inaccurately. Assuming the classification accuracy of feature vectors will be degraded to $90 \%$ due to the various real-time situations, the decision-making precision can be calculated as follows: The probability is $0.1 \%(10 \% \times 10 \% \times 10 \%=0.1 \%)$ if all feature vectors are classified inaccurately. The probability is $2.7 \%((10 \% \times 10 \% \times 90 \%) \times 3=$ $2.7 \%)$ if two feature vectors are classified inaccurately. Therefore, the decisionmaking precision is $97.2 \%(100 \%-2.7 \%-0.1 \%)$.

There are still some challenges in real sEMG-based movement intention understanding and myoelectric control. Most current available myoelectric control systems still use simple open/close control. Robust simultaneous and proportional myoelectric control is still at its early stage. Also, the developed system should be able to adapt to different users. However, this fundamental function is still an open research question.

\section{Conclusions}

The average precision of intact subjects reached $98 \%$ and $91 \%$ upward for amputees by using the proposed classification scheme. However, all the results were generated by using recorded data, the performance of the algorithms and data pre-processing methods are not clear in the real-time situations. Moreover, this approach can adopt to only one subject at a time. Technically the NMF can improve the classification precision as a data pre-treatment method, but the outcome shows that the performance of NMF is unstable. The classification accuracy of amputees can be enhanced 
by using a larger data window size, but the decision-making time is also increased as a consequence.

\section{References}

1. M. Atzori and H. Mller. The ninapro database: A resource for semg naturally controlled robotic hand prosthetics. In 2015 37th Annual International Conference of the IEEE Engineering in Medicine and Biology Society (EMBC), pages 7151-7154, 2015.

2. K. Englehart et al. Classification of the myoelectric signal using time-frequency based representations. Med. Eng. Phys., 21:431-438, 1999.

3. L. J. Hargrove et al. A comparison of surface and intramuscular myoelectric signal classification. IEEE Trans. Biomed. Eng., 54:847-853, 2007.

4. M. Ison, I. Vujaklija, B. Whitsell, D. Farina, and P. Artemiadis. Simultaneous myoelectric control of a robot arm using muscle synergy-inspired inputs from high-density electrode grids. In 2015 IEEE International Conference on Robotics and Automation (ICRA), pages 64696474, 2015.

5. Mark Ison and Panagiotis Artemiadis. The role of muscle synergies in myoelectric control: trends and challenges for simultaneous multifunction control. J. Neural Eng., 11, 2014.

6. T. A. KuiKen et al. Targeted muscle reinnervation for real-time myoelectric control of multifunction artificial arms. JAMA, 301:619-628, 2009.

7. Daniel D. Lee and H. Sebastian Seung. Algorithms for non-negative matrix factorization. In In NIPS, pages 556-562. MIT Press, 2000.

8. A. Manfredo et al. Electromyography data for non-invasive naturally-controlled robotic hand prostheses. Scientific Data., 2014.

9. B. Peerdeman et al. Myoelectric forearm prostheses: state of the art from a user-centred perspective. J. Rehabil. Res. Dev., 48:719-738, 2011.

10. Luka Peternel, Tomoyuki Noda, Tadej Petri?, Ale Ude, Jun Morimoto, and Jan Babi? Adaptive control of exoskeleton robots for periodic assistive behaviours based on emg feedback minimisation. PLOS ONE, 11(2):1-26, 022016.

11. A. D. Roche et al. Prosthetic myoelectric control strategies: a clinical perspective. Curr. Surg. Rep., 2:1-11, 2014.

12. L. H. Smith, T. A. Kuiken, and L. J. Hargrove. Myoelectric control system and task-specific characteristics affect voluntary use of simultaneous control. IEEE Transactions on Neural Systems and Rehabilitation Engineering, 24(1):109-116, 2016. 